\title{
On the Dimorphism and the Pressure-Temperature State Diagram of Gestodene, a Steroidal Progestogen Contraceptive
}

\author{
Ivo B. Rietveld ${ }^{1,2 *}$, Hassan Allouchi ${ }^{3}$ and René Céolin ${ }^{4}$
}

\author{
${ }^{1}$ Normandie Université, SMS-EA3233, Université de Rouen, 76821 Mont Saint Aignan, France \\ ${ }^{2}$ Fac. de Pharmacie, Université Paris Descartes, USPC, 4 av. de l'observatoire, 75006 Paris, France \\ ${ }^{3}$ EA 7502: Synthèse et Isolement de Molécules BioActives. Laboratoire de Chimie Physique, Faculté de Pharmacie, 31, avenue \\ Monge - 37200 Tours, France \\ ${ }^{4}$ LETIAM, EA7357, IUT Orsay, Université Paris Sud, rue Noetzlin, 91405 Orsay, France
}

Keywords: active pharmaceutical ingredient, topological method, crystalline polymorphism

*e-mail: ivo.rietveld@univ-rouen.fr

Gestodene is a steroidal progestogen contraceptive and it exhibits dimorphism [1]. Both polymorphs have an orthorhombic structure with space group $P 2{ }_{1} 2_{1} 2_{1}$. The specific volumes obtained from the unit cell dimensions at room temperature are $0.841 \mathrm{~cm}^{3} \mathrm{~g}^{-1}$ for polymorph I and $0.850 \mathrm{~cm}^{3} \mathrm{~g}^{-1}$ for polymorph II. It demonstrates that form I is denser than form II and its stability should therefore increase with increasing pressure.

The pressure-temperature phase diagram of gestodene is constructed using the temperature and enthalpy of fusion of form I $\left(469.5 \mathrm{~K}, 107 \mathrm{~J} \mathrm{~g}^{-1}\right)$, and those of the endothermic transition from form II to form I $(311 \mathrm{~K}, 8.52$ $\left.\mathrm{J} \mathrm{g}^{-1}\right)$. At ordinary pressure, the sign of the enthalpy is positive indicating an endothermic transition and implying that the two polymorphs are enantiotropically related.

The melting temperature of form II has not been obtained experimentally; however, it can be obtained by calculation using the expression:

$$
T_{I I \rightarrow L}=\frac{\Delta_{I I \rightarrow I} H+\Delta_{I \rightarrow L} H}{\frac{\Delta_{I I \rightarrow I} H}{T_{I I \rightarrow I}}+\frac{\Delta_{I \rightarrow L} H}{T_{I \rightarrow L}}}
$$

This leads to a melting temperature for form II of about $452 \mathrm{~K}$, obviously lower than that of form I, which possesses the stable melting temperature. However, considering the II $\rightarrow$ I transition occurring at $311 \mathrm{~K}$, it can be concluded that form II is the stable form at room temperature. Using the inequality in the specific volumes and in the enthalpy and applying them in the Clapeyron equation, it is shown that the two polymorphs remain enantiotropically related on increasing the pressure since the $\mathrm{dP} / \mathrm{dT}$ slope of the I-II equilibrium line is found to be negative while that of the I-liquid equilibrium is positive (Figure 1) as it is usually the case for a molecular liquid, which has necessarily a higher entropic content than that of the solid state and a lower density than that of the solid state (Water in this respect is a very rare exception due to its higher density in the liquid state than in the solid state).

In addition, it is shown that the heats of dissolution, inferred from solubility measurements, lead to virtually the same value of the heat of transition from polymorph II to polymorph I as the value obtained by differential scanning calorimetry [2].

From a pharmaceutical perspective, it implies that logically form II is the most appropriate form to develop, however, due to the low transition temperature of $311 \mathrm{~K}$ $\left(38{ }^{\circ} \mathrm{C}\right)$ and the fact that form $\mathrm{I}$ is stabilized in relation to form II as a function of pressure, it is important to monitor the polymorphism of this particular drug during processing, because the chances are very high on grinding or compressing the sample that form I could temporarily appear with a possible risk to alter the bioavailability of gestodene in the formulation.

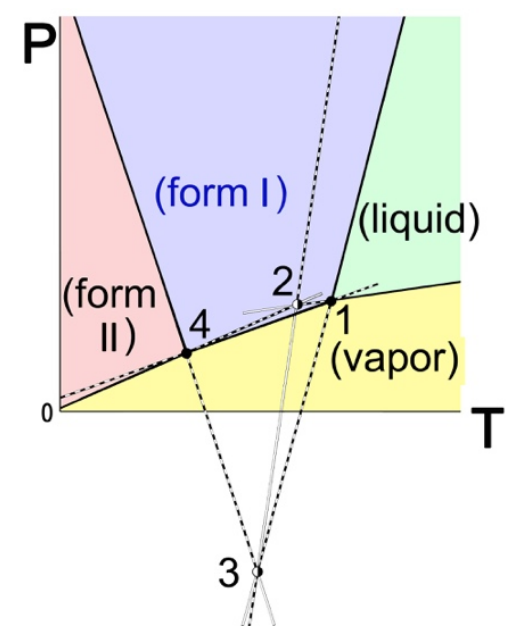

Figure 1. Topological P-T phase diagram for the dimorphism of gestodene with the triple points: $1=$ stable I-liq-vap, $2=$ metastable II-liq-vap, $3=$ metastable I-II-liq, 4 = stable I-II-vap.

[1] F.Z. Stanczyk, D.F. Archer. Contraception 89, 187 (2014).

[2] L. Zhu, L-Y. Wang, Z.L. Sha, Y.F. Wang, L.B. Yang, X.C. Li, X.Y. Zhao, W. Du. Cryst Growth Des 17, 4582 (2017). 